\title{
Possible Drug Candidates for COVID-19
}

\author{
Navan Chauhan
}

March 15, 2020

\begin{abstract}
COVID-19, has been officially labeled as a pandemic by the World Health Organisation. This paper presents cloperastine and vigabatrin as two possible drug candidates for combatting the disease along with the process by which they were discovered. The paper presents the discoveries made by using a connectivity map and the docking configurations used to simulate the docking.
\end{abstract}

\section{Introduction}

COVID-19 has been officially characterised as a pandemic by the World Health Organisation (WHO), therefore a need arises to discover drugs effective in combatting against this virus. In this experiment we try to find possible drug candidates from the existing pool of FDA approved drugs and check their theoretical effectiveness against $6 \mathrm{LU} 7$ and $6 \mathrm{Y} 84$, two protein macromolecules for 2019-nCoV. 6LU7 is the crystal structure of COVID-19 main protease in complex with an inhibitor N3 [1] and 6 Y84 is the COVID-19 main protease with unliganded active site. 2]

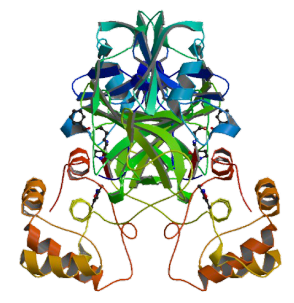

(a) 6LU7

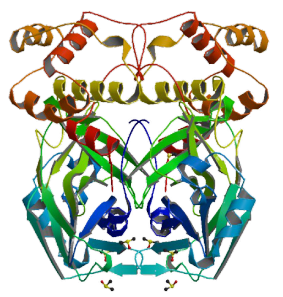

(b) $6 \mathrm{Y} 84$
Figure 1: The target proteins

\section{Background}

\subsection{COVID-19}

On March 11, 2020, COVID-19 was officially characterised as a pandemic by the World Health Organisation. COVID-19 (Also called 2019-nCoV ) is a part of Coronavirus Family (CoV) of viruses which cause various illnesses. The severity of the illnesses is varied, ranging from mild effects to severe, with some cases even being linked to deaths.

\section{$2.2 \quad$ CMap}

A CMap is a large perturbation database which provides opportunities for computational pharmacogenomics and drug design. Comparing transcriptomics profiles 
of targets and drugs already present in the CMap, opens up the door for finding drugs which may already be present in the market. To find possible drug candidates the transcriptomic signatures of the disease and drugs are compared, if the signatures are opposite, this indicates that the drug might inhibit the target disease.

\subsection{Docking}

Molecular Docking is the process by which the binding affinity of a ligand can be predicted for a target molecule. This can help in predicting the bond strength between a protein and a ligand.

\section{$3 \quad$ Experiments}

\subsection{Connectivity Scoring}

The base code for the transcriptomic comparison of the signatures was forked from tinkavidovic/competition. [3] The program which was written in $\mathrm{R}[4]$, was run on an instance of Google Colab as a Jupyter Notebook. The source code and results can be found on the author's GitHub repository navanchauhan/COVID-19. For the transcriptomic comparison, PharmacoGx [5] An $R$ package for for analysis of large pharmacogenomic datasets was used. The normalised disease data analysed was downloaded from the Harmonizome database. [6] [7] [8] 10 drugs each were chosen from the results of the connectivityScore function using the fast gene set enrichment analysis method. After combining the list all the duplicates were deleted. Then the following 14 samples were selected for docking:
- nicergoline (ZINC3873817)

- phenazopyridine (ZINC95483532)

- levomepromazine (ZINC20246)

- vorinostat (ZINC1543873)

- maprotiline (ZINC1530688)

- valproic acid (ZINC3008621)

- resveratrol (ZINC6787)

- nortriptyline (ZINC1530741)

- prochlorperazine (ZINC19796018)

- cloperastine (CHEBI:94448)

- vigabatrin (CHEBI:63638)

- methotrexate (ZINC1529323)

- rescinnamine (ZINC4097185)

- trifluoperazine (ZINC19418959)

\subsection{Docking}

For the docking, we used PyRx [9], a virtual screening software for loading and visualising the SDF files for the ligands and used the AutoDock Vina tool [10] for testing the docking on two target proteins $6 \mathrm{LU} 7$ and 6 Y84 which were downloaded from the Protein Data Bank [11]. The ligands were downloaded from ZINC15. We also docked an extra drug called Remdesivir as it is currently undergoing medical trial. The following configuration was used for docking:

exhaustiveness $=8$

center_x $=-25.9866$

center_y $=12.5889$ 
Table 1: Docking with 6LU7 protein

\begin{tabular}{ll}
\hline Name & Binding Affinity (kcal/mol) \\
\hline Remdesivir & -13.1 \\
cloperastine & -10.4 \\
vigabatrin & -10.2 \\
methotrexate & -6.9 \\
nortriptyline & -6.8 \\
\hline
\end{tabular}

center_z $=59.1569$

size_x $=25$

size_y $=25$

size_z $=25$

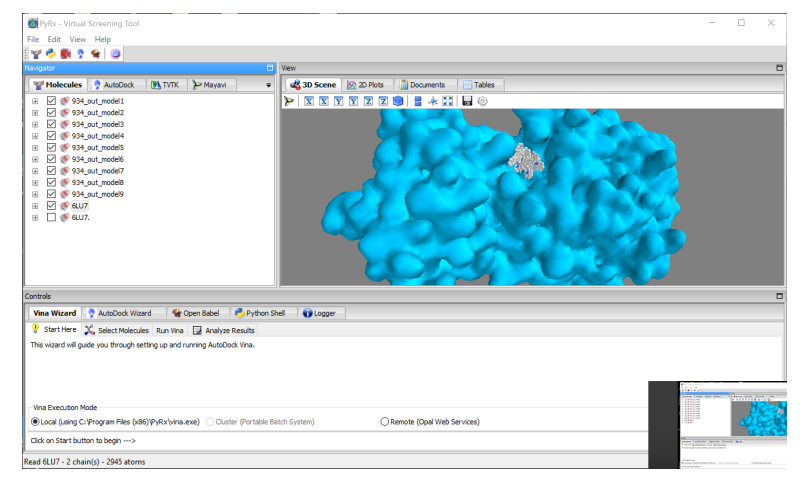

Figure 2: A screenshot of PyRx

\section{Result}

This tables only lists the best binding affinity of top 5 drugs. For the full analysis report, please check the results folder in the repository.

\section{Conclusion}

From the above docking report, we find that along with Remdesivir [12], a drug
Table 2: Docking with 6Y84

\begin{tabular}{ll}
\hline Name & Binding Affinity $(\mathrm{kcal} / \mathrm{mol})$ \\
\hline Remdesivir & -18.6 \\
vigabatrin & -12.1 \\
cloperastine & -10.1 \\
nicergoline & -9.0 \\
phenazopyridine & -8.4 \\
\hline
\end{tabular}

already being tested for its effectiveness against COVID-19, cloperastine and vigabatrin show promise as possible candidates for treating COVID-19. Cloperastine is a cough suppressant that acts on the central nervous system and vigabatrin is a medication used to treat epilepsy. These drugs perform better than the baseline N3 ligand which had an affinity of $7.9 \mathrm{kcal} / \mathrm{mol}$.

\section{Future Work}

The CMap database used for this was last updated in 2015, therefore an analysis with an updated database might help uncover more possible candidates.

The code can be re-run using different variants belonging to the $\mathrm{CoV}$ family to find more drugs.

These drugs need to be clinically tested to check their efficiency against treating COVID-19.

\section{References}

[1] Liu, X., Zhang, B., Jin, Z., Yang, H., Rao, Z. The crystal structure of COVID-19 main protease in complex with an inhibitor N3. To be published. 
[2] Owen, C.D., Lukacik, P., StrainDamerell, C.M., Douangamath, A., Powell, A.J., Fearon, D., BrandaoNeto, J., Crawshaw, A.D., Aragao, D., Williams, M., Flaig, R., Hall, D., McAauley, K., Stuart, D.I., von Delft, F., Walsh, M.A. COVID-19 main protease with unliganded active site. To be published.

[3] Tinka Vidovik. Using Connectivity map data and PharmacoGx $R$ package to find drugs against coronaviruses.

https://github.com/tinkavidovik/competiqiesłoptimization and multithreading.

[4] R Core Team (2018). R: A language and environment for statistical computing. $\mathrm{R}$ Foundation for Statistical Computing, Vienna, Austria.

https://www.R-project.org/.

[5] Smirnov P, Safikhani Z, El-Hachem N, Wang D, She A, Olsen C, Freeman M, Selby H, Gendoo D, Grossman P, Beck A, Aerts H, Lupien M, Haibe-Kains AG, (2016). PharmacoGx: an R package for analysis of large pharmacogenomic datasets.. Bioinformatics (Oxford, England).

[6] Andrew D. Rouillard, Gregory W. Gundersen, Nicolas F. Fernandez, Zichen Wang, Caroline D. Monteiro, Michael G. McDermott, Avi Ma'ayan. The harmonizome: a collection of processed datasets gathered to serve and mine knowledge about genes and proteins. Database (Oxford). 2016 Jul 3;2016.

[7] Barrett, $\mathrm{T}$ et al. (2013). NCBI GEO: archive for functional genomics data sets-update. Nucleic Acids Res. 41:D991-5.
[8] Edgar, R et al. (2002). Gene Expression Omnibus: NCBI gene expression and hybridization array data repository. Nucleic Acids Res. 30:207-10.

[9] Dallakyan S, Olson AJ. SmallMolecule Library Screening by Docking with PyRx. Methods Mol Biol. 2015;1263:243-50.

[10] O. Trott, A. J. Olson. AutoDock Vina: improving the speed and accuracy of docking with a new scoring function, efJournal of Computational Chemistry 31 (2010) 455-461.

[11] Helen M. Berman, John Westbrook, Zukang Feng, Gary Gilliland, T. N. Bhat, Helge Weissig, Ilya N. Shindyalov, Philip E. Bourne. The Protein Data Bank. Nucleic Acids Research, Volume 28, Issue 1, 1 January 2000, Pages 235-242, https://doi.org/10.1093/nar/28.1.235

[12] Kujawski et Al. First 12 patients with coronavirus disease 2019 (COVID-19) in the United States. Preprint. 